\section{Data activism and social change}

Miren Gutiérrez (2018). Data activism and social change. London: Palgrave. Hardcover ISBN: 978-3-319-783185; eBook ISBN: 978-3-319-78319-2

Frente a las desigualdades y las prácticas del dataveillance -como José van Dijck ha llamado a la vigilancia masiva corporativa y gubernamental basada en big data (van Dijck, 2014)-, han surgido formas alternativas de uso de datos que permiten a las personas y organizaciones actuar de forma independiente. Este libro es uno de los mejores ejemplos de un análisis de investigación profundo para describir y explorar cómo son estas prácticas, qué tienen en común y a qué retos se enfrentan.

Data activism and social change aborda cómo las personas y las organizaciones están usando la infraestructura de datos -entendida como el software, hardware y los procesos que permiten convertir los datos en valor- como una herramienta fundamental en sus estrategias. El libro se sitúa en la intersección de dos fenómenos: la interacción entre los datos, la tecnología y las prácticas comunicativas, por un lado, y la participación democrática y la movilización social, por el otro. Es un movimiento que hace uso del big data para el cambio social y la protección de los derechos humanos y del medioambiente. El activismo de datos es una práctica que surge, primero, como resistencia a la dataficación
- entendida como la facultad de traducir en datos cualquier faceta de la vida- y la vigilancia masiva y que pone en el eje de su investigación en la infraestructura de datos para el cambio social, la asistencia humanitaria, la defensa de los derechos humanos y el medioambiente.

Es desafiante reseñar la complejidad y originalidad de esta obra, considerada uno de los 12 mejores libros a nivel mundial sobre activismo de datos según Book Authority ${ }^{1}$. El libro se basa en treinta entrevistas semiestructuradas a expertos y expertas, activistas y representantes del mundo de la investigación, que dependen de la infraestructura de datos para su trabajo, se definen como periodistas y activistas de datos, o usan los datos con un propósito social. Estas personas incluyen desde un epidemiólogo que ve en los datos la posibilidad de empoderar a las personas en la medicina social y una periodista que ha manejado el mayor filtrado de datos de la historia a un arquitecto que usa datos ciudadanos y participativos para diseñar espacios públicos. Asimismo, se basa en los resultados de la observación de más de cuarenta organizaciones y casos que hacen de los datos el centro de su actividad. Se trata de comunidades, organizaciones e individuos que están cartografiando conflictos, generando narrativas alternativas, movilización y soluciones a problemas sociales.

El objetivo de este trabajo es describir un fenómeno nuevo el activismo de datos-y analizar sus mecanismos y estructuras sobre una amplia base de casos reales y entrevistas con la comunidad pionera de expertos y expertas que lo practican. Está dividido en cinco partes. Primero, a modo de contexto, se describen los usos de la infraestructura de datos en varios sectores. Después, sobre la base de los casos y las entrevistas, se define y explora el activismo proactivo de datos como una práctica social. A continuación, se profundiza en la plataforma de humanitarismo digital Ushahidi como una práctica que ilustra el activismo de datos. Ushahidi está especializada en apoyar los esfuerzos humanitarios en casos de crisis, guerras, violencia, emergencias y desastres. Finalmente, se propone un modelo para un activismo de datos efectivo.

Frente a la opacidad y cerrazón con que las corporaciones niegan el acceso a datos que han recolectado gratuitamente de sus usuarios, consumidores o clientes, el libro aporta un análisis de las estrategias y prácticas de organizaciones e individuos que obtienen o generan tales datos y, así, contribuyen al activismo social. Las principales contribuciones del libro son las dos tipologías realizadas des-

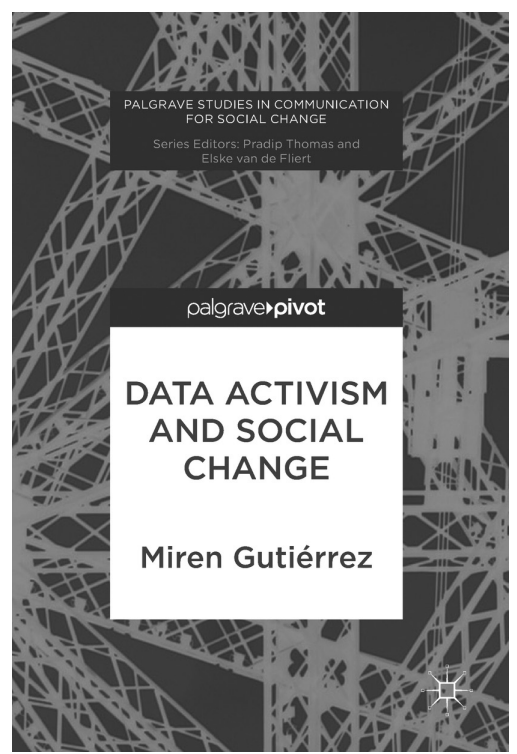


de dos ópticas diferentes li.e. “a qué se dedican los y las activistas de datos" y "de dónde sacan los datos"). Las clasificaciones se ofrecen como una herramienta heurística, para observar y vertebrar otros casos, que está siendo utilizada como la base de otros estudios (e.g. Briones 2019). Primero, los y las activistas de datos pueden ser quienes transfieren habilidades tecnológicas, quienes catalizan y hacen posibles proyectos de datos, quienes producen periodismo de datos como una forma de activismo, y quienes se dedican al activismo de datos como actividad central. Una segunda taxonomía clasifica las iniciativas desde la perspectiva de las maneras en que las y los activistas adquieren datos de forma alternativa, que van desde la obtención de datos a partir de denunciantes y filtrados len inglés whistleblower) y el acceso a datos públicos y abiertos a la creación de plataformas y comunidades para recabar datos ciudadanos y la puesta en marcha de mecanismos, redes y aparatos que recogen datos (e.g. drones comunitarios).

Ushahidi es un ejemplo del tercer tipo de organización, ya que se basa en el crowdsourcing de datos ciudadanos, que puede ser un proceso poderoso. Para demostrarlo, Gutiérrez dedica un espacio considerable en su obra a estudiar la plataforma Ushahidi. El mapa del terremoto de Haití en 2010, utilizando la plataforma Ushahidi, abordó vacíos de información clave en el período inicial antes de que las agencias humanitarias estuvieran operativas en el terreno. A través de la plataforma se suministraron datos geolocalizados a pequeñas organizaciones no gubernamentales más bien invisibilizadas y brindaron información situacional rápida con alto grado de exactitud a la ciudadanía para la toma de decisiones en medio del desastre. El mapa de Haití marcó la transición hacia un cambio de paradigma ante la presencia de emergencias o catástrofes naturales, dando lugar al humanitarismo digital.

Entre los múltiples ejemplos discutidos por la autora se cuentan también "Liquid Traces", de Forensic Architecture, organización que empleó señales satelitales, de calor y de radar, entre otras tecnologías de vigilancia, para documentar que la negligencia fue la causa de la denegación de ayuda a 72 migrantes huyendo de Libia en marzo de 2011. A pesar de que diversas autoridades costeras de distintos países del borde Mediterráneo recibieron las señales de auxilio, nadie fue en su rescate y apenas 9 personas sobrevivieron. WeRobotics, en tanto, ha ayudado a comunidades de Nepal a analizar y mapear su vulnerabilidad ante diversas consecuencias del cambio climático, como las avalanchas.

La autora identifica también once características que definen a organizaciones que trabajan con datos desde el activismo. Entre ellas destaca el trabajo en alianzas, pues, a menudo, las causas exceden con mucho los recursos disponibles, por ejemplo, en casos de violaciones a derechos humanos o en extensas bases de datos. Otra cualidad es que los y las activistas de datos frecuentemente desarrollan mapas como herramienta para el análisis, la coordinación, la comunicación y la movilización. Históricamente, los mapas han sido objetos dotados de conocimiento, poder y autoridad, y el apogeo de la cartografía digital, la tecnología móvil, el crowdsourcing de datos y los sistemas de información geográfica hacen que los mapas sean más poderosos aún que los mapas convencionales. Esto ocurre en coincidencia con un interés creciente en el mapeo de las crisis, una práctica que combina las capacidades de la geoweb con la asistencia humanitaria y las campañas. En ese sentido, en manos de las personas y organizaciones adecuadas, los mapas han sido una forma de contrapoder político. El empleo de mapas es tan útil que la autora los considera una variedad específica del activismo de datos: el geoactivismo. InfoAmazonia, una organización dedicada a asuntos ambientales y derechos humanos en la región amazónica, es un ejemplo de organización especializada en la visualización de datos geolocalizados.

Otra característica es que los y las activistas de datos generalmente son flexibles en mezclar métodos y herramientas de diversas disciplinas o actores. Numerosas organizaciones son híbridas porque cruzan las líneas que suelen separar el periodismo, la investigación, el humanitarismo y el activismo, pero también porque combinan repertorios de acción de diferentes áreas. Un ejemplo es Los vagabundos de chatarra, un proyecto periodístico que incluye cómics, un libro, mapas interactivos, vídeos y un sitio web para contar las historias de personas que sobrevivieron la crisis económica en Barcelona recogiendo y vendiendo chatarra ( $\mathrm{Ca}$ rrion, 2016). 
La variedad y complejidad de los numerosos casos analizados por la autora hacen que, a veces, la lectura obligue a tender puentes entre espacios y casos cuya conexión no es fácil de seguir. En todo caso, este libro aborda un asunto contemporáneo de primer orden: cómo las personas y la sociedad civil revierten procesos tecnológicos y políticos para proponer sus propias narrativas y soluciones, y supone una lectura obligatoria para especialistas en comunicación y activismo social.

El texto es una contribución a los estudios críticos de datos, en la línea de Nancy Gitelman (Gitelman, 2013) y Tom Boellstorff (Boellstorff, 2013). Sabemos que los datos y los algoritmos no "hablan" por sí mismos y que no son neutrales. Los datos no pueden ser "crudos"; los datos y metadatos están "cocinados" en procesos que no surgen de modo natural, sino que siempre son construidos y elaborados en base a unos intereses. Es decir, los big data no son recursos naturales, necesarios y automáticos, sino recursos culturales que deben ser generados, tratados y transformados. El hecho de que la infraestructura de datos se utilice para causas nobles, como en el activismo de datos, no elimina los sesgos y las asimetrías presentes en las bases de datos, los algoritmos y los procesos de transformación. El libro no soslaya este asunto, pero se centra en que, incluso empleando tecnologías imperfectas, este tipo de activismo obtiene resultados.

\section{Nota}

1 Sobre la base de menciones públicas, recomendaciones, evaluaciones y ventas, entre otros indicadores, Book Authority elabora listas y recomendaciones sobre libros de no ficción. Ver https://bookauthority.org/books/new-social-activismbooks.

\section{Referencias}

Boellstorff, T. (2013). "Making Big Data, in Theory." First Monday 18 (10). http:// firstmonday.org/article/ view/4869/3750.

Briones, A. (2019). “Disclose to Tell. A Data Design Framework for Alternative Narratives." PhD Dissertation, Milan, Italy: Politecnico di Milano. https://densitydesign.org/research/ disclose-to-tell-a-data-design-framework-for-alternative-narratives/.

Carrion, J. (2016). Los Vagabundos de La Chatarra. Barcelona: Norma.

Van Dijck, J. 2014. “Datafication, Dataism and Dataveillance: Big Data between Scientific Paradigm and Ideology." Surveillance \& Society 12 (2): 197-208.

Gitelman, L. (ed.) (2013). Raw Data Is an Oxymoron. Cambridge, Massachusetts, London: The MIT Press.

\section{María Pilar Rodríguez Pérez}

Universidad de Deusto, Bilbao, España.

maria.pilarवdeusto.es 